\title{
Nível de evidência das tecnologias de cuidado de enfermagem na angioplastia de membros inferiores
}

\author{
Viviane Soares ${ }^{1}$, Juliana Balbinot Reis Girondi ${ }^{2}$, Karina Silveira de Almeida Hammerschmidt ${ }^{3}$, \\ Lúcia Nazareth Amante ${ }^{4}$, Darla Lusia Ropelato Fernandez ${ }^{5}$, Luciara Fabiane Sebold ${ }^{6}$
}

\footnotetext{
${ }^{1}$ Enfermeira, Mestre em Enfermagem. Florianópolis, SC, Brasil. E-mail: vivi_zimba@hotmail.com.

${ }^{2}$ Enfermeira, Doutora em Enfermagem. Professor Titular da Universidade Federal de Santa Catarina. Florianópolis, SC, Brasil. E-mail: juliana.balbinot@ufsc.br.

${ }^{3}$ Enfermeira, Doutora em Enfermagem. Professor Adjunto da Universidade Federal de Santa Catarina. Florianópolis, SC, Brasil. E-mail: karina.h@ufsc.br.

${ }^{4}$ Enfermeira, Doutora em Enfermagem. Professor Associado da Universidade Federal de Santa Catarina. Florianópolis, SC, Brasil. E-mail: luciamante@gmail.com.

${ }^{5}$ Enfermeira, Mestre em Enfermagem. Discente do Programa de Pós-Graduação em Enfermagem, nível Doutorado, da Universidade Federal de Santa Catarina. Professor Substituto da Universidade Federal de Santa Catarina. Florianópolis, SC, Brasil. E-mail: darla.fernandez@ufsc.br.

${ }^{6}$ Enfermeira, Doutora em Enfermagem. Professor Adjunto da Universidade Federal de Santa Catarina. Florianópolis, SC, Brasil. E-mail: fabiane.sebold@ufsc.br.
}

Recebido: 18/07/2015.

Aceito: 30/06/2016.

Publicado: 21/12/2016.

\section{RESUMO}

Objetivou-se descrever as produções científicas sobre tecnologias de cuidado de enfermagem ao paciente adulto submetido à angioplastia de membros inferiores. Revisão Sistemática da Literatura com busca nas bases de dados eletrônicas: LILACS, PUBMED, Web of Science, SciELO, além de Google Acadêmico. Foram analisados e classificados 12 artigos conforme os níveis de evidência. Todos os estudos qualitativos foram classificados com baixo grau de recomendação e dos estudos quantitativos apenas um apresentou alto grau de recomendação. A pesquisa apresentou estudos com o mais baixo nível de evidência científica, além da escassez de pesquisas e o frágil embasamento científico no qual os cuidados perioperatórios de enfermagem ao paciente submetido à angioplastia de membros inferiores vêm sendo desenvolvidos. Por conseguinte, há falta de informações e formação deficiente culminando no despreparo para atender os pacientes, compreender e liderar esse serviço de alta complexidade consoante aos princípios de segurança do paciente.

Descritores: Hemodinâmica; Assistência Perioperatória; Enfermagem Baseada em Evidências.

\section{INTRODUÇÃO}

No setor de hemodinâmica são realizados procedimentos terapêuticos do aparelho circulatório servindo aos propósitos de especialidades médicas como cirurgia vascular, cardiologia e gastroenterologia. Nesse serviço é comum o tratamento de doenças 
vasculares periféricas, afecções caracterizadas pela redução do fluxo sanguíneo proveniente de obstrução por placas ateroscleróticas, trombos, êmbolos, válvulas venosas incompetentes ou por redução na ação de bombeamento dos músculos circunvizinhos ${ }^{(1)}$.

Doenças vasculares são tratadas especialmente por procedimento de angioplastia transluminal percutânea, que possui algumas vantagens sobre a cirurgia convencional, quais sejam: tempo de internação reduzido, menor risco de complicações, dor de pequena intensidade e menor custo $^{(2)}$. Estudos têm evidenciado eficácia no tratamento e vantagens para a recuperação dos pacientes submetidos e esse tipo de procedimento $^{(3)}$.

A isquemia crítica, por exemplo, é uma doença vascular que pode levar à amputação de membros inferiores e é importante causa de mortalidade ${ }^{(4-5)}$. Nesses casos, um número crescente de centros está adotando primeiramente o tratamento por abordagem endovascular, por angioplastia, obtendo sucesso significativo, como o estudo realizado pela Universidade da Califórnia no Davis Medical Center, que analisou pacientes que se submeteram a procedimentos endovasculares para tratamento de isquemia crítica, entre 2006 e 2012 e obteve taxas favoráveis de salvamento do membro afetado ${ }^{(6)}$.

O setor de hemodinâmica é considerado serviço de alta complexidade e requer uma equipe de enfermagem atuante, capacitada, competente, eficiente, ágil e atualizada. Além de atividades assistenciais, o enfermeiro no serviço de hemodinâmica desenvolve funções de liderança, gerenciamento de materiais e de pessoal, o que exige agilidade e tomada de decisões rápidas. Em meio a esse processo, compete ao enfermeiro o dimensionamento de pessoal, supervisão e treinamento da equipe, controle dos materiais médico-hospitalares utilizados em cada procedimento, bem como o conhecimento de condutas em relação ao reprocessamento dos materiais utilizados ${ }^{(7)}$.

Em meio à alta tecnologia que envolve o procedimento de angioplastia, a enfermagem deve se fazer valer de instrumentos metodológicos para embasar a assistência prestada. Para tanto, o cuidado de enfermagem baseado em evidências científicas relevantes, propicia eficácia e segurança, já que é obtido por meio de estudos e pesquisas em bases de dados confiáveis ${ }^{(8)}$.

A identificação destas tecnologias de cuidado embasadas em evidências científicas se faz necessária para implementação de cuidados de enfermagem condizentes com as necessidades da população. As doenças cardiovasculares são um tema amplamente debatido atualmente devido ao aumento das taxas de morbidade e mortalidade e pelo fato de serem doenças evitáveis e tratáveis. Além disso, são foco de políticas públicas específicas no território brasileiro, como a Política Nacional de Atenção Cardiovascular de Alta Complexidade e outras relacionadas à prevenção destas como, a Política Nacional de Promoção da Saúde, Política Nacional de Atenção Básica, Política Nacional de Atenção Integral à Hipertensão Arterial Sistêmica e ao Diabetes Mellitus. Consoante, as tecnologias de cuidados devem estar articuladas a esta rede de cuidados para que os portadores de doenças cardiovasculares possam ser melhor assistidos, tendo garantia de acesso, cobertura e atendimento em uma rede de cuidados organizada em todos os níveis de atenção à saúde.

Nesse contexto, cabe averiguar quais níveis de evidência têm embasado as tecnologias de cuidados 
empregadas pela enfermagem a pacientes submetidos à angioplastia de membros inferiores, no período perioperatório. A necessidade de investigação sobre esse nível de atenção, especificamente, se faz necessária uma vez que estes cuidados ainda são baseados na experiência clínica e empírica dos profissionais que prestam assistência a este tipo de paciente. Estes cuidados poucas vezes são baseados em evidências científicas e foi justamente esta lacuna que motivou a buscar argumentos científicos que justifiquem os cuidados prestados com foco neste procedimento.

Assim, os objetivos do presente estudo foram: descrever as produções científicas disponíveis na literatura que englobem as tecnologias de cuidado de enfermagem ao paciente adulto submetido à angioplastia de membros inferiores.

\section{MÉTODO}

Trata-se de uma Revisão Sistemática de Literatura de natureza descritiva ${ }^{(9)}$. Previamente a este estudo se verificou a existência de Revisões Sistemáticas sobre o tema proposto na Biblioteca Cochrane (The Cochrane Database of Systematic Reviews), mas não foram encontradas revisões nesta área.

A pergunta de pesquisa que originou esta Revisão foi: "Quais são as produções científicas existentes sobre as tecnologias de cuidado de Enfermagem ao paciente submetido à angioplastia de membros inferiores"?

A busca dos estudos primários foi realizada nas bases de dados, PubMed/MEDLINE (Public MEDLINE), Web of Science, Scientific Eletronic Library Online (SciELO) e Literatura Latino-Americana e do Caribe em Ciências da Saúde (LILACS). Além das bases pesquisou-se no Google Acadêmico ${ }^{\circledR}$.

Para cada base de dados foi estabelecida uma estratégia de busca baseando-se na pergunta de pesquisa, conforme Quadro 1. Para a composição da estratégia de busca, utilizou-se os termos do MeSH (Medical Subject Headings Terms), que foram aplicados na busca PUBMED/MEDLINE. Para a LILACS empregou-se o DECs (descritores em ciências da saúde). Utilizou-se também os operadores booleanos (delimitadores) representados pelos termos AND, OR e NOT. As palavras-chave foram pesquisadas em português e inglês. O período da busca foi de agosto de 2014 a maio de 2016, por dois autores de forma independente.

Os critérios de inclusão foram estudos: a) quantitativos, qualitativos ou quali-quantitativos que apresentassem em seu título ou no resumo a temática relacionada às tecnologias de cuidado de enfermagem no período perioperatório de angioplastia de membros inferiores; b) com seres humanos na faixa etária maior ou igual a 18 anos, submetidos à angioplastia de membros inferiores; c) disponíveis nas línguas inglesa, portuguesa ou espanhola. Os estudos incluídos não tiveram como filtro o ano de publicação, visto que é uma revisão sistemática e o tema ainda pouco estudado na área da Enfermagem.

Foram critérios de exclusão: normas técnicas, manuais, leis, resoluções, editoriais, cartas, comentários, resumo de anais e publicações duplicadas. 
Quadro 1: Estratégias de busca por bases de dados.

\begin{tabular}{|c|c|}
\hline $\begin{array}{l}\text { Base de } \\
\text { Dados }\end{array}$ & Estratégia de busca \\
\hline PUBMED & $\begin{array}{l}\text { ("angioplasty"[MeSH Terms] OR "angioplasty"[All Fields] OR "Endovascular treatment"[All Fields] OR "endovascular } \\
\text { procedures"[MeSH Terms] OR "endovascular procedures"[All Fields] OR "endovascular procedure"[All Fields) AND } \\
\text { ("nursing"[Subheading] OR "nursing"[Title/Abstract] OR "nursing"[MeSH Terms] OR "nursing care"[All Fields] OR } \\
\text { "nursing care"[MeSH Terms] OR "nurses"[MeSH Terms] OR "nurses"[All Fields] OR "nurse"[All Fields]) AND } \\
\text { (English[lang] OR Portuguese[lang] OR Spanish[lang]) }\end{array}$ \\
\hline $\begin{array}{l}\text { Web of } \\
\text { Science }\end{array}$ & $\begin{array}{c}\text { Tópico: (angioplast* OR Endovascular treatment OR endovascular procedure*) AND Tópico: (nursing OR nursing } \\
\text { care OR nurse OR nurses) }\end{array}$ \\
\hline SCIELO & $\begin{array}{l}\text { (angioplast\$ OR endovascular\$ OR "tratamento endovascular" OR "tratamentos endovasculares" OR } \\
\text { "procedimento endovascular" OR"procedimentos endovasculares" OR "Endovascular treatment" OR "endovascular } \\
\text { procedures" OR "endovascular procedure") AND (nursing OR enfermagem OR enfermeir\$ OR nurse\$) }\end{array}$ \\
\hline LILACS & $\begin{array}{l}\text { (mh:("Angioplastia" OR "Procedimentos Endovasculares") OR tw:("Angioplastia" OR "Procedimentos } \\
\text { Endovasculares" OR "Procedimento Endovascular" OR "tratamento endovascular" OR "tratamentos } \\
\text { endovasculares")) AND (mh:("enfermagem" OR "enfermeiras" OR "enfermeiros" OR "cuidados de enfermagem" } \\
\text { OR "Avaliação em Enfermagem" OR "Diagnóstico de Enfermagem" OR "Enfermagem Baseada em Evidências" OR } \\
\text { "Enfermagem Cardiovascular" OR "Enfermagem Perioperatória") OR tw:("enfermagem" OR enfermeirł OR } \\
\text { "cuidados de enfermagem" OR "Avaliação em Enfermagem" OR "Diagnóstico de Enfermagem" OR "Enfermagem } \\
\text { Baseada em Evidências" OR "Enfermagem Cardiovascular" OR "Enfermagem Perioperatória")) }\end{array}$ \\
\hline
\end{tabular}

A seleção dos estudos baseou-se nos critérios de inclusão e na pergunta de pesquisa no formato acrônimo $\mathrm{PICO}^{(10)}$ onde: onde $\mathrm{P}=$ População/Participantes: Pacientes adultos submetidos à angioplastia de membros inferiores; I= Intervenções: Cuidados de Enfermagem na angioplastia de membros inferiores e o uso de Tecnologias de Cuidados de Enfermagem aos pacientes submetidos à angioplastia de membros inferiores; $C=$ Comparações: não há; $O=$ Outcomes/Desfecho: Cuidados e Tecnologias de cuidado de Enfermagem eficazes no período perioperatório de angioplastia de membros inferiores.

Nesta Revisão Sistemática (RS) foram identificados inicialmente 3.824 estudos. A Figura 1 representa o fluxograma do processo de seleção e inclusão das produções científicas. 


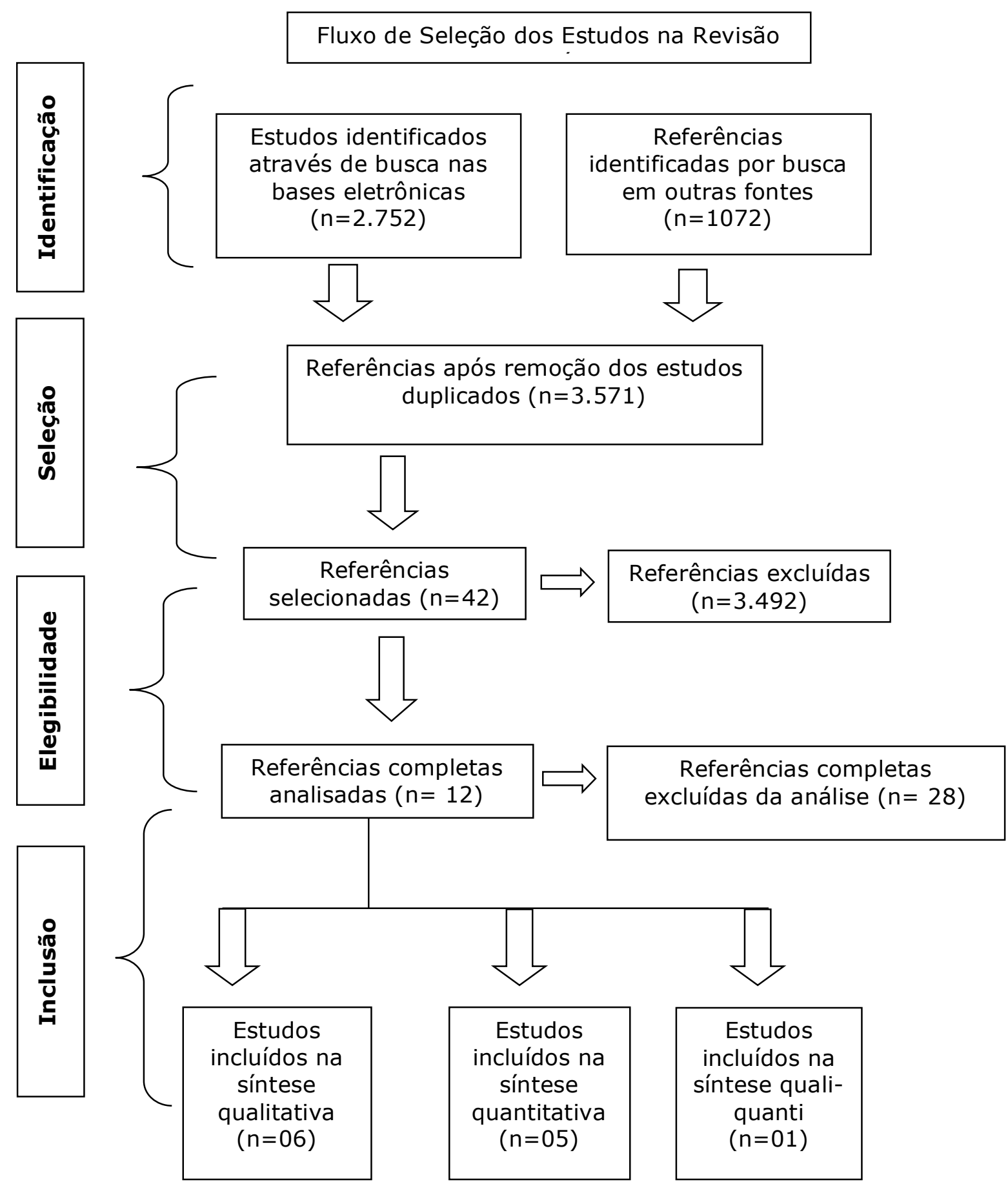

Figura 1: Fluxograma de seleção de estudos, de acordo com os critérios pré-estabelecidos.

Para a extração dos dados foi elaborada uma tabela com as características dos estudos incluídos, que permitiu a comparabilidade das variáveis destes, o país/ano e origem de publicação, o objetivo e os métodos empregados. Os resultados foram apresentados na forma descritiva, sendo os estudos classificados de acordo com o nível de evidência ${ }^{(11)}$.

\section{RESULTADOS}

Nesta RS foram analisados 12 estudos que atenderam aos critérios de inclusão e exclusão do protocolo de pesquisa. 0 Quadro 2 apresenta a caracterização destes e a classificação dos níveis de evidência, onde se 
pode perceber que a maioria das publicações ocorreu entre o ano de 2006 a 2016, apresentando um número maior a partir de 2011. A publicação mais antiga identificada sobre o tema é do ano de 2006 e a mais recente é do ano de 2016. 
Quadro 2: Síntese dos artigos quanto a identificação, base de dados, país de origem de publicação, objetivo, resultados, método, resultados e nível de evidência.

\begin{tabular}{|c|c|c|c|c|c|}
\hline Autor/Ano & Base de Dados & $\begin{array}{c}\text { País de } \\
\text { origem da } \\
\text { publicação }\end{array}$ & Objetivo & Resultados & $\begin{array}{l}\text { Nível de } \\
\text { Evidência }\end{array}$ \\
\hline \multicolumn{6}{|c|}{ ESTUDOS COM MÉTODO QUALITATIVO } \\
\hline $\begin{array}{l}\text { (12) Kasthuri R, } \\
\text { Karunaratne D, } \\
\text { Andrew H, Sumner J, } \\
\text { Chalmers N. / } 2007 \text {. }\end{array}$ & PubMed/MEDLINE & Reino Unido & $\begin{array}{l}\text { Auditar a segurança da intervenção arterial } \\
\text { periférica, dos casos de Angioplastia Periférica, } \\
\text { por enfermeiros para aconselhamento, } \\
\text { cuidados e acompanhamento desses pacientes } \\
\text { pós alta hospitalar. }\end{array}$ & $\begin{array}{l}\text { Em pacientes encaminhados para a intervenção vascular periférica } \\
\text { eletiva, a hemostasia pós-procedimento foi realizada com } \\
\text { compressão manual. Após } 3 \text { horas no leito (deitado na cama), os } \\
\text { pacientes foram mobilizados e tiveram alta em } 5 \text { horas. Os pacientes } \\
\text { foram contatados por telefone no próximo dia útil para auditar as } \\
\text { complicações. Das } 183 \text { cirurgias eletivas realizadas em } 2 \text { anos, em } \\
\text { cinco paciente ( } 2 \% \text { ) houve necessidade de retorno pois complicações } \\
\text { locais na região de virilha, sendo que num dos casos o paciente tinha } \\
\text { falso aneurisma. Nos demais quatro casos não houve necessidade de } \\
\text { tratamentos adicionais. }\end{array}$ & VI \\
\hline $\begin{array}{l}{ }^{(13)} \text { Vieira LC, Contrin } \\
\text { LM, Rol JL, Conte HD, } \\
\text { Lima ARS, Castro } \\
\text { EDR, et al./ } 2009 .\end{array}$ & Google Acadêmico & Brasil & $\begin{array}{l}\text { Identificar as dificuldades e necessidades da } \\
\text { equipe de Enfermagem em serviços de } \\
\text { hemodinâmica e angiografia no país. }\end{array}$ & $\begin{array}{l}\text { O estudo identificou a dificuldade do enfermeiro no processo de } \\
\text { gerenciamento e liderança no setor de Hemodinâmica. Da mesma } \\
\text { forma esta dificuldade foi percebida durante a execução de diversas } \\
\text { atividades, desde as mais simples até as mais complexas. A } \\
\text { responsabilidade de supervisão do enfermeiro de hemodinâmica é } \\
\text { ampla, como gerenciar materiais de alto custo e liderança da equipe. }\end{array}$ & VI \\
\hline $\begin{array}{l}{ }^{(14)} \text { Krauzer IM, } \\
\text { Brocardo D, Scarsi } \\
\text { T./2011. }\end{array}$ & Google Acadêmico & Brasil & $\begin{array}{l}\text { Identificar o perfil de saúde dos clientes } \\
\text { assistidos no Centro de Hemodinâmica e } \\
\text { aplicar o Processo de Enfermagem, de acordo } \\
\text { com Callista Roy. }\end{array}$ & $\begin{array}{l}\text { O estudo identificou o perfil de } 52 \text { clientes atendidos no Centro de } \\
\text { Hemodinâmica de um hospital privado brasileiro. Os resultados } \\
\text { obtidos foram o perfil de saúde dos clientes atendidos, de modo a } \\
\text { oferecer características sobre o tipo de clientela, por meio da } \\
\text { identificação da faixa etária; do local de moradia; estado civil; da } \\
\text { escolaridade; ocupação; dos fatores de risco para doenças } \\
\text { cardiovasculares; Índice de Massa Corporal e dos tipos mais } \\
\text { frequentes de intervenções cirúrgicas. Depois foi delineado o } \\
\text { Processo de Enfermagem, onde foram elencados os diagnósticos de } \\
\text { Enfermagem no período pós-operatório e o percentual de incidência } \\
\text { nos clientes: Ansiedade relacionada à morte (100\%), Risco de dor } \\
\text { aguda (85\%), Integridade da pele prejudicada (75\%), Perfusão } \\
\text { tissular ineficaz, Perfusão tissular ineficaz, Risco de infecção (24\%) e } \\
\text { Risco de inibição da cascata de coagulação (16\%). }\end{array}$ & VI \\
\hline
\end{tabular}




\begin{tabular}{|c|c|c|c|c|c|}
\hline Autor/Ano & Base de Dados & $\begin{array}{l}\text { País de } \\
\text { origem da } \\
\text { publicação }\end{array}$ & Objetivo & Resultados & $\begin{array}{l}\text { Nível de } \\
\text { Evidência }\end{array}$ \\
\hline $\begin{array}{l}\text { (15) Bomfim FMTS, } \\
\text { Lima SG, Victor EG./ } \\
2013 .\end{array}$ & Google Acadêmico & Brasil & $\begin{array}{l}\text { Avaliar a prevalência e as condições do } \\
\text { reprocessamento de cateteres de } \\
\text { hemodinâmica na cidade do Recife, PE - Brasil. }\end{array}$ & $\begin{array}{l}\text { Foram avaliados quatro hospitais da rede pública e seis da rede } \\
\text { privada em uma capital brasileira. Todas as instituições reprocessam } \\
\text { cateteres. Apenas um deles realiza todas as etapas do } \\
\text { reprocessamento na própria instituição. Na maioria dos hospitais, os } \\
\text { cateteres são reutilizados, em média, quatro vezes. Observou-se } \\
\text { significativa divergência nas respostas fornecidas pelos participantes } \\
\text { do estudo, o que refletiu na falta de padronização das técnicas } \\
\text { utilizadas no reprocessamento. A maioria dos profissionais já } \\
\text { detectou fraturas e tortuosidades nos cateteres reprocessados. A } \\
\text { preparação enzimática é o produto mais empregado para a limpeza } \\
\text { dos cateteres. Em relação à limpeza do lúmen destes dispositivos } \\
\text { não houve uniformidade entre as respostas fornecidas pelos } \\
\text { investigados. Embora a maioria dos profissionais utilize a água da } \\
\text { torneira para o enxágue dos cateteres, as técnicas utilizadas são } \\
\text { bastante diversificadas. Testes para verificação de materiais } \\
\text { pirogênicos não são executados em todos os materiais } \\
\text { reprocessados. }\end{array}$ & VI \\
\hline $\begin{array}{l}{ }^{(16)} \text { Maher P. J Vasc } \\
\text { Nurs./2014. }\end{array}$ & PubMed/MEDLINE & Irlanda & $\begin{array}{l}\text { Examinar a viabilidade de alta no mesmo dia } \\
\text { após angioplastia, com ênfase na obtenção de } \\
\text { resultados seguros paciente. }\end{array}$ & $\begin{array}{l}\text { O estudo explora como os avanços nas tecnologias e técnicas } \\
\text { endovasculares contribuíram para tornar a alta no mesmo dia uma } \\
\text { opção cada vez mais viável. A pré-admissão clínica dos pacientes } \\
\text { desenvolvidas por enfermeiros especializados facilita a seleção do } \\
\text { paciente de forma apropriada e segura, onde os pacientes em risco } \\
\text { de problemas pós-procedimento podem ser identificados de forma } \\
\text { eficaz com base em critérios clínicos pré-definidos. Com base nestas } \\
\text { condutas, alta hospitalar no mesmo dia após angioplastia para } \\
\text { doença vascular periférica é uma opção segura e viável. }\end{array}$ & VI \\
\hline
\end{tabular}




\begin{tabular}{|c|c|c|c|c|c|}
\hline Autor/Ano & Base de Dados & $\begin{array}{c}\text { País de } \\
\text { origem da } \\
\text { publicação }\end{array}$ & Objetivo & Resultados & $\begin{array}{l}\text { Nível de } \\
\text { Evidência }\end{array}$ \\
\hline $\begin{array}{l}{ }^{(17)} \text { Wann-Hansson C, } \\
\text { Wennick A. BMC } \\
\text { Nurs./2016. }\end{array}$ & PubMed/MEDLINE & Suécia & $\begin{array}{l}\text { Elucidar como os pacientes com doença } \\
\text { arterial periférica comunicam o seu } \\
\text { conhecimento sobre a sua doença e } \\
\text { tratamentos. }\end{array}$ & $\begin{array}{l}\text { A pesquisa identificou que o curto espaço de tempo gasto com os } \\
\text { pacientes com doença arterial periférica requer orientação } \\
\text { inovadora na prática clínica para atender às necessidades dos } \\
\text { indivíduos, independentemente de o paciente entender sua doença } \\
\text { arterial periférica. Durante as discussões sobre fatores de risco, } \\
\text { observou-se o tabagismo como um importante fator de risco, que } \\
\text { desencadeou sentimento de culpa. É necessário para os pacientes } \\
\text { consultar fontes de informação para gerir as suas vidas diárias. Nesse } \\
\text { processo o enfermeiro é essencial para o desenvolvimento de ações } \\
\text { de educação em saúde. }\end{array}$ & VI \\
\hline \multicolumn{6}{|c|}{ ESTUDOS COM MÉTODO QUANTITATIVO } \\
\hline $\begin{array}{l}{ }^{(18)} \text { Akopian G, Katz } \\
\text { SG./ } 2006 .\end{array}$ & $\underline{\text { PubMed/MEDLINE }}$ & $\begin{array}{l}\text { Estados } \\
\text { Unidos }\end{array}$ & $\begin{array}{l}\text { Avaliar a possibilidade de alta hospitalar no } \\
\text { mesmo dia após angioplastia periférica }\end{array}$ & $\begin{array}{l}\text { Nos } 97 \text { pacientes submetidos à angioplastia periférica, a artéria } \\
\text { femoral superficial foi o local mais frequente de intervenção (47\%) e } \\
\text { estes pacientes receberam alta hospitalar no mesmo dia de } \\
\text { realização do procedimento. Nessa população estudada, um } \\
\text { paciente foi internado por um grande hematoma no local da punção } \\
\text { necessitando de transfusão de sangue, dois pacientes para a } \\
\text { observação de um hematoma menor no local da punção, um por dor } \\
\text { no peito, e um para observação de bradicardia transitória. O tempo } \\
\text { médio para a mobilização pós procedimento foi de } 1,4 \text { horas, e o } \\
\text { tempo médio de alta foi de } 2,8 \text { horas pós intervenção. Nenhuma } \\
\text { morte ou internação não planejada ocorreu em tempo igual ou } \\
\text { superior a } 30 \text { dias de intervenção no hospital. }\end{array}$ & VI \\
\hline $\begin{array}{l}{ }^{(19)} \text { Gioppato S, } \\
\text { Castello HJ Jr, } \\
\text { Conforti TB, } \\
\text { Gonçalves SLP, } \\
\text { Morais FGS, } \\
\text { Cantarelli MJC. / } \\
2011 .\end{array}$ & Google Acadêmico & Brasil & $\begin{array}{l}\text { Avaliar a relação custo-efetividade da retirada } \\
\text { imediata da bainha femoral e oclusão com } \\
\text { dispositivo de oclusão vascular AngioSeal TM } \\
\text { comparativamente à hemostasia por } \\
\text { compressão manual e/ou mecânica após } \\
\text { intervenções endovasculares. }\end{array}$ & $\begin{array}{l}\text { Comparado à compressão manual e/ou mecânica como técnicas de } \\
\text { hemostasia em procedimentos terapêuticos endovasculares o } \\
\text { dispositivo AngioSeal TM não houve diferença específicas em relação } \\
\text { ao sucesso da hemostasia sem complicações. O custo individual do } \\
\text { tratamento das complicações relacionadas à hemostasia por } \\
\text { compressão foi maior que o custo do dispositivo de oclusão. }\end{array}$ & $\mathrm{VI}$ \\
\hline
\end{tabular}




\begin{tabular}{|c|c|c|c|c|c|}
\hline Autor/Ano & Base de Dados & $\begin{array}{l}\text { País de } \\
\text { origem da } \\
\text { publicação }\end{array}$ & Objetivo & Resultados & $\begin{array}{l}\text { Nível de } \\
\text { Evidência }\end{array}$ \\
\hline $\begin{array}{c}{ }^{(20)} \text { Covello CM. / } \\
2011 .\end{array}$ & Google Acadêmico & Brasil & $\begin{array}{l}\text { Comparar as taxas de complicações vasculares } \\
\text { no sítio de acesso femoral em idosos } \\
\text { submetidos à hemostasia, mecânica ou clássica } \\
\text { da artéria femoral após procedimentos } \\
\text { percutâneos. }\end{array}$ & $\begin{array}{l}\text { Foram randomizados } 110 \text { pacientes, destes } 54 \text { submetidos a } \\
\text { compressão manual e } 56 \text { a compressão mecânica. As taxas de } \\
\text { complicações vasculares maiores foram na fase hospitalar e no } \\
\text { seguimento de sete dias. No seguimento clínico de sete dias foi } \\
\text { observado que a taxa total de complicações vasculares maior no } \\
\text { grupo da compressão manual (64\%) bem como a taxa de pacientes } \\
\text { com complicações vasculares nesta técnica (48\%). A taxa de } \\
\text { complicações vasculares menores não foi diferente para os grupos } \\
\text { nos dois períodos. }\end{array}$ & II \\
\hline $\begin{array}{l}\text { (21) Albayati MA, } \\
\text { Gohel MS, Patel SR, } \\
\text { Riga CV, Cheshire } \\
\text { NJW, Bicknell CD./ } \\
2011 .\end{array}$ & Web of Science & Reino Unido & $\begin{array}{l}\text { Investigar falhas na segurança do paciente } \\
\text { submetidos à procedimentos vasculares e } \\
\text { endovasculares para orientar a qualidade e a } \\
\text { segurança nas futuras intervenções. }\end{array}$ & $\begin{array}{l}\text { Em } 66 \text { procedimentos vasculares observados foram identificadas } \\
1145 \text { falhas que comprometerem a segurança do paciente durante } \\
\text { os procedimentos. Destas } 23,5 \% \text { das falhas foram relacionadas a } \\
\text { equipamentos, } 21 \% \text { falhas relacionadas à comunicação da equipe, } \\
3,6 \% \text { falhas técnicas e } 5,4 \% \text { falhas psicomotoras. }\end{array}$ & $\mathrm{VI}$ \\
\hline $\begin{array}{l}{ }^{(22)} \text { Eufrásio S, Sousa P, } \\
\text { Oliveira N, Gonçalves } \\
\text { F, Alves G, Rodrigues } \\
\text { H, et al. / } 2013 .\end{array}$ & Scielo & Portugal & $\begin{array}{l}\text { Registrar, avaliar e quantificar a dose de } \\
\text { radiação distribuída pela equipe cirúrgica pela } \\
\text { exposição à radiação dispersa, emitida por um } \\
\text { aparelho portátil de radioscopia com arco } \\
\text { cirúrgico (arco em C), durante a utilização em } \\
\text { bloco operatório. }\end{array}$ & $\begin{array}{l}\text { O estudo constatou como nível máximo de exposição a radiação } \\
\text { dispersa pela equipe assistente à altura ao solo de } 120 \mathrm{~cm} \text {. Os níveis } \\
\text { de radiação foram inferiores quando utilizados os meios de proteção } \\
\text { radiológica. }\end{array}$ & $\mathrm{VI}$ \\
\hline \multicolumn{6}{|c|}{ ESTUDOS COM MÉTODO MISTO } \\
\hline $\begin{array}{l}\text { (23) Lundén M, } \\
\text { Lundgren SM, } \\
\text { Persson LO, Lepp M. / } \\
\text { 2013. }\end{array}$ & PubMed/MEDLINE & $\begin{array}{l}\text { Estados } \\
\text { Unidos }\end{array}$ & $\begin{array}{l}\text { Identificar pacientes que são } \\
\text { predominantemente ansiosos ou calmos antes } \\
\text { do tratamento com angioplastia transluminal } \\
\text { percutânea e explorar as razões para estes } \\
\text { sentimentos. }\end{array}$ & $\begin{array}{l}\text { O estudo mensurou o estado emocional dos pacientes pré- } \\
\text { angioplasta percutnea evidenciando que a maioria (69\%) deles sentiu } \\
\text { bastante calma antes da angioplastia. Este sentimento se deu em } \\
\text { virtude da confiança na equipe de saúde cuidadora, bem como na } \\
\text { capacidade desta de prever os riscos cirúrgicos. Os pacientes que se } \\
\text { sentiram ansiosos antes do procedimento tiveram essa causa } \\
\text { relacionada ao medo de um resultado negativo e se sentiram } \\
\text { inseguros em relação as opções de tratamento. }\end{array}$ & $\mathrm{VI}$ \\
\hline
\end{tabular}


Das 12 produções conclui-se que estas concentram-se no Brasil (cinco), seguido pelos Estados Unidos (duas), Reino Unido (duas), Portugal (uma), Portugal, Irlanda (uma), Suécia (uma).

Em relação à fonte pesquisada, um artigo foi encontrado na base de dados SciELO, um na base de dados Web of Science, cinco artigos na PUBMED e cinco produções na ferramenta Google Acadêmico ${ }^{\circledR}$. A base de dados LILACS não trouxe nenhuma produção pertinente. Dessa forma, o idioma mais publicado foi o português (seis), seguido do inglês (cinco). Não foram encontradas produções no idioma espanhol.

\section{DISCUSSÃO}

Os 12 estudos selecionados para essa revisão sistemática foram explorados na sua totalidade de acordo com a temática proposta. A partir dos achados encontrados nos estudos, optou-se por criar categorias para uma melhor discussão. Estas categorias baseiam-se na metodologia dos estudos e delas emergiram três grupos: a) estudos qualitativos; b) estudos quantitativos; c) estudo quali-quantitativo (método misto).

\section{Estudos qualitativos}

Foram encontrados quatro produções científicas nesta categoria ${ }^{(12-17)}$.

Primeiramente destaca-se um estudo descritivo realizado em 2007 com 183 pacientes que realizaram procedimento de intervenção arterial periférica e objetivou averiguar a segurança no procedimento realizado. Foi feito contato telefônico para avaliar o acompanhamento dos enfermeiros durante o período perioperatório, onde a maioria dos pacientes considerou a estratégia importante. Esse acompanhamento pelo enfermeiro é realizado no período pré-operatório via telefone, onde estes fornecem informações sobre o procedimento que será realizado. Além disso, o paciente recebe via correio um folheto com informações relativas à intervenção. Na ocasião da alta, os pacientes recebem do enfermeiro presencialmente as informações pertinentes, além do telefone de contato do serviço e do enfermeiro de plantão para os contatos necessários. No pós-operatório, via contato telefônico, dos 183 casos, apenas dois pacientes (1\%) tiveram complicações e cinco tiveram no dia seguinte a alta hospitalar edema em região inguinal e retornaram para avaliação. O estudo evidencia que o acompanhamento dos casos por enfermeiros é importante, pois além de satisfazer o paciente, reduz custos hospitalares e evita problemas de falta de leitos para a internação no caso de procedimentos eletivos ${ }^{(12)}$.

Outra pesquisa qualitativa foi o estudo exploratório-descritivo realizado em 2009 em 30 cidades brasileiras, o que contempla 17 estados, para identificar as dificuldades e necessidades da equipe de enfermagem que atua nos serviços de hemodinâmica e angiografia destes locais. Dentre as dificuldades encontradas estão: a falta de experiência na área, a escassez de literatura na área voltada para a enfermagem, o gerenciamento da unidade, a fragilidade de programas de educação permanente e a dificuldade de gerenciamento de tempo para verificação do reprocessamento de materiais. Referencia ainda, as atribuições assistenciais desenvolvidas por enfermeiros. Evidencia que as dificuldades de troca de informações e a formação deficiente levam ao despreparo para atender os pacientes, de compreender e 
liderar esse serviço de alta complexidade ${ }^{(13)}$.

Corroborando esse achado, outro estudo destaca que a Enfermagem deve embasar o cuidado no Processo de Enfermagem a fim de identificar fatores de risco e atuar na prevenção das complicações decorrentes de procedimentos endovasculares. Ao estudar 52 pacientes de um hospital particular de Chapecó/SC identificou-se como fatores de risco: hipertensão arterial sistêmica, idade acima de 60 anos, obesidade e sedentarismo. Dos fatores de risco identificados nesta amostra elencaram-se os diagnósticos de enfermagem: ansiedade relacionada à morte (100\%), risco de dor aguda $(85 \%)$, integridade da pele prejudicada (75\%), risco de perfusão tissular ineficaz tipo renal (42\%), risco de infecção (24\%) e risco de inibição da cascata de coagulação $(16 \%)^{(14)}$. Portanto, a implementação do Processo de Enfermagem no cuidado destes pacientes é premissa essencial para a efetivação de um cuidado seguro e de qualidade.

Em relação ao reprocessamento de cateteres de hemodinâmica foram entrevistados 92 profissionais de enfermagem atuantes em 10 serviços na cidade de Recife/PE. Verificou-se que todos estes serviços reprocessam os cateteres utilizados nos procedimentos hemodinâmicos em média há cinco anos, $70 \%$ das instituições reprocessam aproximadamente 20 dispositivos ao dia e estes cateteres são reutilizados em média quatro vezes. O estudo destaca que não há uma padronização das técnicas de reprocessamento de cateteres de hemodinâmica e que nenhum dos serviços pesquisados segue um protocolo validado que oriente as etapas no reprocessamento. Indica ainda, que a vigilância na qualidade do reprocessamento não deve ser restrita aos dispositivos utilizados, mas ao registro das ocorrências adversas ao paciente ${ }^{(15)}$.

As evidências científicas dos estudos qualitativos, de forma geral, discorrem sobre as atividades desenvolvidas por enfermeiros, que vão desde atividades assistenciais, perpassando as gerenciais, de educação em saúde e supervisão. Enfatizam essa gama de atividades, mas destacando enquanto principais atribuições do enfermeiro: uma pré-avaliação do paciente planejada e sistematizada ${ }^{(16)}$, o monitoramento do paciente no pós-procedimento e na alta hospitalar, o fornecimento de informações/orientações no período perioperatório; além da detecção precoce de complicações pós-procedimento, mediante identificação prévia dos riscos cirúrgicos e da implementação do Processo de Enfermagem. Para o desempenho de tais atribuições as tecnologias de cuidado emergentes são: acompanhamento e monitoramento do paciente por contato telefônico ${ }^{(12)}$; elaboração e aplicação do processo de enfermagem durante o cuidado prestado no período perioperatório de angioplastia ${ }^{(13)}$; avaliação clínica pré-admissional feita pelo enfermeiro para detecção precoce de riscos cirúrgicos e alta precoce ${ }^{(16)}$; educação em saúde com base nas necessidades do paciente ${ }^{(17)}$; vigilância na qualidade do reprocessamento de cateteres utilizados na hemodinâmica e acompanhamento das ocorrências adversas ${ }^{(12-15)}$. Apesar de importantes tecnologias de cuidados para a Enfermagem, todos os estudos qualitativos que compuseram a amostra desta RS foram classificados como nível de evidência VI, demonstrando que são estudos com baixo grau de recomendação.

As várias tecnologias de cuidados que subsidiam o fazer do enfermeiro em hemodinâmica, enfatizando a responsabilidade deste pela assistência integral ao paciente e no desenvolvimento de atividades gerenciais, de ensino e pesquisa ${ }^{(22-26)}$. Em relação à educação de pacientes submetidos à angioplastia transluminal 
percutânea os enfermeiros devem se tornar conhecedores do estilo de vida do paciente para implementar um plano de cuidados individualizado no pré e pós intervenção ${ }^{(27)}$.

\section{Estudos quantitativos}

Essa categoria foi composta por cinco estudos ${ }^{(18-22)}$, sendo que quatro deles se enquadram na classificação VI, ou seja, estudos com baixo grau de recomendação. Apenas um estudo se destacou enquanto nível de evidência II por se tratar de ensaio clínico randomizado-controlado, ou seja, estudo experimental desenvolvido com seres humanos que visou o conhecimento do efeito das técnicas de hemostasia pósangioplastia, configurando-se como uma das ferramentas mais poderosas para obtenção de evidências clínicas para a prática.

Um estudo de 2013, feito por meio de uma simulação registrou, avaliou e quantificou a dose de radiação reproduzindo as condições habituais em que ocorrem os procedimentos endovasculares, na equipe cirúrgica e no paciente, que estão expostos à radiação dispersa. Identificou que nos doentes, procedimentos prolongados com a utilização de magnificação eletrônica podem causar queimaduras na pele e aumentam consideravelmente a taxa de dose de radiação incidente no doente. Como meio para diminuir a quantidade de radiação incidente no doente e consequente radiação dispersa, preconiza a aproximação do intensificador de imagem ao paciente, além do controle eficaz do tempo de procedimento, modo de fluoroscopia e magnificação. Destaca ainda a importância da monitoração dos valores de radiação e que o uso adequado dos equipamentos de proteção radiológica pode permitir condições de segurança para a equipe de saúde e para o paciente ${ }^{(16)}$.

Já um estudo realizado em 2011 investigou 66 procedimentos endovasculares por nove meses e encontrou 1.847 eventos adversos e 1.145 falhas identificadas, mediante aplicação de check list de verificação de segurança cirúrgica da Organização Mundial da Saúde. As falhas mais comuns identificadas foram: 23,5\% em equipamentos (indisponibilidade, falhas na configuração, gestão do espaço e falhas do aparelho); $21 \%$ na comunicação entre cirurgião e circulante, anestesista e cirurgião; $9 \%$ em técnicas. A pesquisa descreve que poucas falhas graves foram identificadas, mas houve o acúmulo de falhas menores que aconteceram em momentos mais críticos do procedimento. Destaca que a sala de cirurgia vascular é um ambiente complexo, dinâmico e abriga uma equipe multidisciplinar envolvendo a troca de grande quantidade de informações e tarefas cirúrgicas de alta complexidade. Logo, a prevenção do erro cirúrgico representa um grande desafio, mas deste problema ${ }^{(17)}$.

Uma publicação de 2006 descreve sobre o uso de protocolo para alta hospitalar no mesmo dia pósangioplastia eletiva em 97 pacientes por um período de 27 meses. A pesquisa aponta que a angioplastia periférica em pacientes com claudicação pode ser realizada com segurança em ambulatório, com significativa redução de custos. A conclusão aponta que a alta no mesmo dia pós-angioplastia periférica é segura e eficaz, portanto é considerada padrão outro de atendimento. Para ter este desfecho, apesar de ser resultados de aplicação de um protocolo médico, o artigo aponta alguns aspectos inerentes ao cuidado de enfermagem, 
como: uso de medicamentos pré e trans-procedimento, tempo de mobilização pós-procedimento e tempo de compressão manual no local de realização da intervenção ${ }^{(18)}$. No entanto, na realidade brasileira, grande parte dos procedimentos endovasculares exige permanência hospitalar até o dia seguinte à realização do procedimento, pois a maioria destes envolve situações clínicas e/ou anatômicas complexas ${ }^{(19)}$.

Dois estudos dessa categoria avaliaram a questão das técnicas de hemostasia pós-angioplastia. Em um deles foi estudado os resultados, complicações e custos envolvidos no uso de um dispositivo de oclusão vascular comparado à compressão manual/mecânica pós-intervenção endovascular. Foram analisados 407 pacientes submetidos à intervenções endovasculares de 2006 a 2009, num hospital de Campinas/SP. A conclusão é que a técnica de hemostasia por compressão manual e/ou mecânica quando bem realizada é tão efetiva quanto à hemostasia com uso de oclusão vascular, porém com um custo significativamente menor. Ou seja, o dispositivo de oclusão vascular não se mostrou mais seguro ou com melhor relação custoefetividade quando comparado à compressão manual ou mecânica ${ }^{(19)}$. O segundo estudo comparou as taxas de complicações vasculares no sítio de acesso femoral em 110 idosos, em virtude da hemostasia mecânica (com dispositivo grampo tipo " $\mathrm{C}$ ") ou clássica após procedimentos percutâneos. A pesquisa concluiu que não houve diferença entre estas taxas de complicações vasculares entre as técnicas hemostáticas ${ }^{(20)}$.

Nessa categoria as evidência científicas apontam para tecnologias de cuidados emergentes, quais sejam: adotar estratégias para redução da quantidade de radiação incidente no paciente durante o procedimento de angioplastia ${ }^{(16)}$; monitorar os valores de radiação e adequação dos meios de proteção radiológica ${ }^{(16)}$; implantar um protocolo de segurança pré-procedimentos endovasculares ${ }^{(17)}$; implantar a lista de verificação de segurança cirúrgica ${ }^{(17)}$; padronizar a técnica de compressão hemostática pós-procedimento de angioplastia ${ }^{(19-20)}$.

\section{Estudo quali-quantitativo (método misto)}

Nessa categoria foi contemplado apenas um estudo americano, onde foram entrevistados 42 pacientes submetidos a procedimentos endovasculares e aplicada a escala de Likert a fim de identificar os pacientes ansiosos ou calmos antes do procedimento e explorar as razões para estes sentimentos. As razões listadas para estar calmo foram estar seguros, ter boas expectativas quanto ao procedimento e segurança nos cuidadores (médicos e enfermeiros). Em contrapartida, a ansiedade esteve relacionada ao desespero e apreensão sobre o procedimento, medo de um resultado negativo e insegurança quanto às opções de tratamento. A pesquisa aponta que estudos sobre os sentimentos dos pacientes pré-angioplastia são escassos.

O estudo apesar de ser classificado quanto ao nível de evidência em VI traz algumas importantes tecnologias de cuidado a serem empregadas nesse contexto, quais sejam: conhecer as percepções do paciente pré-procedimento; estar aberto ao diálogo e escuta qualificada; utilizar terminologia adequada durante orientações; fornecer orientações pré-procedimento (escritas, orais e por vídeo) sobre o procedimento, fatores de risco, local de realização da intervenção e tratamentos; utilizar musicoterapia antes 
do procedimento; incentivar a criação de grupos de pacientes; utilizar abordagem individualizada e acolhedora antes da angioplastia ${ }^{(21)}$.

\section{CONCLUSÃO}

A revisão apresentou estudos com o mais baixo nível de evidência científica, a escassez de estudos e o frágil embasamento científico no qual os cuidados perioperatórios de enfermagem ao paciente submetido à angioplastia de membros inferiores vêm sendo desenvolvidos. Por conseguinte, há falta de informações e formação deficiente culminando no despreparo para atender os pacientes, compreender e liderar esse serviço de alta complexidade.

Como limitação deste estudo destaca-se, a heterogeneidade entre os artigos pesquisados, principalmente, em relação aos sujeitos de pesquisa investigados e intervenções o que impossibilitou uma análise comparativa entre os achados.

\section{REFERÊNCIAS}

1. Jude EB, Eleftheriadou I, Tentolouris N. Peripheral arterial disease in diabetes - a review. Diabetic Medicine. [Internet]. 2010 [cited 2015 jun 10];27(1):4-14. Available from: http://www.ncbi.nlm.nih.gov/pubmed/20121883 2. Von Ristow A, Massiére BV, Vescovi A. Oclusões arteriais crônicas infrainguinais: Tratamento cirúrgico. In: Brito CJ. Cirurgia Vascular: Cirurgia endovascular Angiologia. 3. ed. Rio de Janeiro: Revinter; 2014.

3. Lo RC, Bensley RP, Dahlberg SE, Matyal R, Hamdan AD, Wyers M, et al. Presentation, treatment, and outcome differences between men and women undergoing revascularization or amputation for lower extremity peripheral arterial disease. Journal of Vascular Surgery. [Internet]. 2014 [cited 2015 jun 10]; 59(2):409-418. Available from: http://www.ncbi.nlm.nih.gov/pubmed/24080134

4. Varu VN, Hogg ME, Kibbe MR. Critical limb ischemia. Journal of Vascular Surgery. [Internet]. 2010 [cited 2015 jun 20];51(1):230-241. Available from: http://www.sciencedirect.com/science/article/pii/S0741521409017832

5. McCoach CE, Armstrong EJ, Singh S, Javed U, Anderson D, Yeo KK, et al. Gender-related variation in the clinical presentation and outcomes of critical limb ischemia. Vasc Med. [Internet] 2013 [cited 2015 jun 15];18(1):19-26. Available from: http://www.ncbi.nlm.nih.gov/pubmed/23439776

6. Singh GD, Armstrong EJ, Yeo KK, Singh S, Westin GG, Pevec WC, et al. Endovascular recanalization of infrapopliteal occlusions in patients with critical limb ischemia. J. Vasc. Surg. [Internet] 2014 [cited 2015 jun 15];59(5):1300-1307. Available from: http://www.ncbi.nlm.nih.gov/pubmed/24393279

7. Linch GFC, Guido LA, Fantin SS. Enfermeiros de unidades de hemodinâmica do Rio Grande do Sul: perfil e satisfação profissional. Texto e contexto-enermagem. [Internet] 2010 [acesso em: 15 jun 2015];19(3):488-495. Disponível em: http://www.scielo.br/pdf/tce/v19n3/a10v19n3.pdf

8. Karino ME, Felli VEA. Enfermagem baseada em evidências: avanços e inovações em Revisões Sistemáticas. Revista Ciência, Cuidado e Saúde. [Internet] 2012 [acesso em: 20 jun 2015];11(suplem):11-15. Disponível em: http://periodicos.uem.br/ojs/index.php/CiencCuidSaude/article/view/17048/pdf

9. Galvão TF; Pereira M. Revisões sistemáticas da literatura: passos para sua elaboração. Epidemiol Serv Saude. [Internet] 2014 [acesso em: 20 jun 2015];23(1):183-4. Disponível em:

http://scielo.iec.pa.gov.br/pdf/ess/v23n1/v23n1a18.pdf.

10. Nobre M, Bernardo W. Prática clínica baseada em evidências. Rio de Janeiro: Elsevier; 2006.

11. Melnyk BM, Fineout O (editors). Evidence-based practice in nursing and healthcare: a guide to best practice. 2 . ed. Philadelphia: Wolters Kluwer Health/Lippincott Williams and Wilkins; 2011 [cited 2015 jan 10]. Available from: http://libguides.ohsu.edu/content.php?pid=249886\&sid=2079582.

12. Kasthuri R, Karunaratne H, Andrew H, Summer J, Chalmers N. Day-case peripheral angioplasty using nurse-led 
admission, discharge, and follow-up procedures: arterial closure devices are not necessary. Clinical Radiology. [Internet]. 2007 [cited 2014 ago 10];62:1202-1205. Available from:

http://www.sciencedirect.com/science/article/pii/S0009926007002838\#.

13. Vieira LC, Contrin LM, Rol JL, Del-Conte H, Lima ARS, Castro EDR, et al. Dificuldades e Necessidades da Equipe de Enfermagem em Serviços de Hemodinâmica e Angiografia. Arq Ciênc Saúde. [Internet] 2009 [acesso em: 10 ago 2014];16(1):21-25. Disponível em: http://repositorio-racs.famerp.br/racs ol/vol-16-1/ID_300.pdf.

14. Krauzer IM, Brocardo D, Scarsi T. A metodologia de Callista Roy aplicada em clientes submetidos à intervenção hemodinâmica. R. Enferm. Ufsm. [Internet]. 2011 [acesso em 10 ago 2014];1(2):183-193. Disponível em:

http://cascavel.ufsm.br/revistas/ojs-2.2.2/index.php/reufsm/article/view/2513/1631.

15. Bomfim FMTS, Lima SG, Victor EG. Análise do Reprocessamento de Cateteres de Hemodinâmica em uma Capital Brasileira. Rev Bras Cardiol. [Internet]. 2013 [acesso em: 13 set 2014];26(1):33-39. Disponível em:

http://www.rbconline.org.br/artigo/analise-do-reprocessamento-de-cateteres-de-hemodinamica-em-uma-capitalbrasileira/.

16. Maher P. Same-day discharge after angioplasty for peripheral vascular disease: is it a safe and feasible option? 2014 [acesso em: 20 mai 2016];32(3):119-24. Disponível em: http://www.ncbi.nlm.nih.gov/pubmed/16730157.

17. Wann-Hansson C, Wennick A. How do patients with perifheral arterial disease communicate their knoweledge about their illness and treatments? A qualitative study. 2016 [acesso em: 10 mai 2016];4: 15-29. Disponível em: http://www.ncbi.nlm.nih.gov/pubmed/27147906.

18. Akopian G, Katz SG. Peripheral angioplasty with same-day discharge in patients with intermittent claudication. J Vasc Surg. Chicago. [Internet]. 2006 [cited 2014 nov 12];44(1):115-118. Available from:

http://www.ncbi.nlm.nih.gov/pubmed/16730157.

19. Gioppato S, Castello Jr HJ, Conforti TB, Gonçalves SLP, Morais FGS, Cantarelli MJC. Análise da Relação CustoEfetividade do Dispositivo de Oclusão Vascular AngioSealTM comparado à Compressão Manual e/ou Mecânica após Intervenções Endovasculares. Revista Brasileira de Cardiologia Invasiva. [Internet]. 2011 [acesso em: 12 nov 2014];19(2):189-193. Disponível em: http://www.rbci.org.br/detalhe artigo.asp?id=547.

20. Covello CM. Complicações vasculares no sítio de acesso femoral em idosos após procedimentos percutâneos: comparação entres as técnicas hemostáticas de compressão manual e mecânica com dispositivo grampo C. 2011. [dissertação]. Recife: Faculdade de Enfermagem/UFPE; 2011 [acesso em: 15 ago 2014]. 108 p. Disponível em: http://repositorio.ufpe.br/bitstream/handle/123456789/1643/arquivo2889 1.pdf?sequence=1.

21. Albayati MA, GoehI MS, Patel SR, Riga CV, Cheshire NJW, Bicknell CD. Identification of Patient Safety Improvement Targets in Successful Vascular and Endovascular Procedures: Analysis of 251 hours of Complex Arterial

Surgery. European Journal Of Vascular And Endovascular Surgery. [Internet]. 2011 [cited 2014 out 14]; 41(6):795802. Available from: http://www.ejves.com/article/S1078-5884(11)00055-4/fulltext.

22. Eufrásio S, Sousa $P$, Oliveira N, Gonçalves $F$, Alves $G$, Rodrigues $H$, et al. Risco da exposição à radiação ionizante durante procedimentos endovasculares. Angiol Cir Vasc. [Internet]. 2013 [acesso em: 13 set 2014];3(9):84-89.

Disponível em: http://www.elsevier.pt/pt/revistas/angiologia-e-cirurgia-vascular-388/artigo/risco-da-exposicao-aradiac-ao-ionizante-durante-90252535.

23. Lunden M, Lundgren SM, Persson LO, Leep M. Patients' experiences and feelings before undergoing peripheral percutaneous transluminal angioplasty. J Vasc Nurs. [Internet]. 2013 [cited 2014 nov 12];31(4):158-164. dez. 2013. Available from: http://www.jvascnurs.net/article/S1062-0303(13)00032-0/fulltext.

24. Girlene RC, Saraí BC, Luciane LS, Thiego RS, Adriana KAF, Francielzo FL. Atuação do enfermeiro no serviço de hemodinâmica: uma revisão integrativa. R. Interd. [Internet] 2014 [acesso em: 29 mai 2016];7(3):157-64. Disponível em: file:///C:/Users/Usuario/Downloads/468-1063-1-PB\%20(1).pdf

25. Linch GFC, Guido LA, Pitthan LO, Umann J. Unidades de Hemodinâmica: a produção do conhecimento. Rev. Gaúcha Enferm. [Internet]. 2009 [acesso em: 15 jan 2015];30(4):742-749. Disponível em:

http://www.scielo.br/scielo.php?script=sci_arttext\&pid=S1983-14472009000400022.

26. Mardikar HM, Mukherjee D. Current Endovascular Treatment of Peripheral Arterial Disease. Prog Cardiovasc Nurs. [Internet] 2007 [cited 2015 jan 15];22(1):31-37. Available from:

http://www.ncbi.nlm.nih.gov/pubmed/17342004.

27. Yellen EA. Synergy and peripheral percutaneous transluminal angioplasty. J Vasc Nurs. [Internet]. 2007 [cited 2015 jan 15];25(1):7-11. Available from: http://www.jvascnurs.net/article/S1062-0303(06)00121-X/fulltext. 